\title{
Integrating Behavior in Life-History Theory: Allocation versus Acquisition?
}

\section{Laskowski, Kate}

2021-02

Laskowski , K , Moiron , M \& Niemelä , P T 2021 , ' Integrating Behavior in Life-History

Theory: Allocation versus Acquisition? ' , Trends in Ecology \& Evolution , vol. 36 , no. 2 , pp.

132-138 . https://doi.org/10.1016/j.tree.2020.10.017

http://hdl.handle.net/10138/340570

https://doi.org/10.1016/j.tree.2020.10.017

cc_by_nc_nd

acceptedVersion

Downloaded from Helda, University of Helsinki institutional repository.

This is an electronic reprint of the original article.

This reprint may differ from the original in pagination and typographic detail.

Please cite the original version. 
Integrating behavior in life-history theory: allocation versus acquisition?

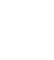

3

4

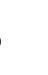

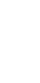

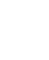

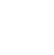

9

10

1

\title{
Highlights:
}

\author{
${ }^{2}$ CEFE, Univ Montpellier, CNRS, EPHE, IRD, Univ Paul Valéry Montpellier 3, Montpellier, France
}

${ }^{3}$ Behavioral Ecology, Department of Biology, Ludwig-Maximillians University of Munich, Planegg-

${ }^{4}$ Organismal and Evolutionary Biology Research Programme, University of Helsinki, Helsinki, Finland

* Corresponding author: Laskowski, K.L. (klaskowski@ucdavis.edu)

*Twitter contact: @KateLaskowski, @MariaMoironn, @PetriNiemel2

Keywords: Pace-of-life syndrome hypothesis, personality, life-history theory, trade-offs

- Current theory predicts that among-individual variation in behavior is maintained by variation in how individuals resolve life-history trade-offs.

- Individuals that exhibit 'fast' phenotypes are expected to allocate more into current reproduction and acquire more resources to fuel this investment, while 'slow' phenotypes, with lower acquisition, are predicted to allocate more into future reproduction.

- Thus, current theory is assuming the presence of behaviorally-mediated among-individual variation in both resource allocation and resource acquisition, simultaneously.

- Here we highlight how explicit considerations of whether behavior is more closely linked to variance in resource acquisition or resource allocation can improve predictions about the observed correlations between behavior and life-history traits, drawing general implications for life-history theory. 


\section{Abstract}

Central theories explaining the maintenance of individual differences in behavior build on the assumption that behavior mediates life-history trade-offs between current and future reproduction. However, current empirical evidence does not robustly support this assumption. This mismatch might be because current theory is not clear about the role of behavior in an individual's allocation versus acquisition of resources, hindering its empirical testing. The relative importance of allocation compared to acquisition is a key feature of classic life-history theory but appears to have been lost in translation in recent developments of life-history theory involving behavior. We argue that determining the relative balance between variation in resource allocation and acquisition, and behavior's role in this, will help build more robust and precise predictions.

\section{Modern "pace-of-life syndrome" framework: where are we standing?}

For the last two decades, understanding the existence of among-individual variation (see Glossary) in behavioral expression (i.e. 'animal personality') has been a main focus of behavioral, evolutionary and animal ecology $[1,2]$. One of the central theories explaining the existence and maintenance of these individual differences in behavior relies on the assumption that behavior mediates a trade-off between resource acquisition and mortality risk: individuals expressing 'riskier' behaviors (i.e. higher aggression, boldness, exploration, activity) are able to acquire and hold more resources, compared to less risky individuals, but in doing so expose themselves to higher mortality risk [2-4]. This modern 'pace-of-life syndrome' (POLS) framework has generated testable predictions about patterns of trait correlations where 'fast' phenotypes are expected to invest heavily in current reproduction (e.g. faster growth, earlier reproduction, higher metabolic rates) at the expense of survival, whereas 'slow' phenotypes will prioritize survival and future reproduction. The modern POLS framework has inspired a whole generation of empiricists that put these theories to the test over the last decade, including the use of similar frameworks in human life-history research [5,6, and sometimes abused 7]. However, the accumulating empirical effort has revealed that the predicted patterns of correlations among behavior and other traits are not well supported [8-10]. 
apparent mismatch between theoretical predictions and empirical evidence. We argue that the specific role that behavior plays as a mediator of life-history trade-offs and specifically whether, and what role it plays in influencing an individual's resource allocation compared to its resource acquisition needs to be further refined in a new POLS framework.

\section{Relative balance of allocation to acquisition: lost in translation?}

The classic life-history trade-off between current and future reproduction predicts that the resources an individual allocates to current reproduction comes at the expense of resources available for future reproduction leading to a negative association between the two [11]. There is strong empirical support for this at the among-species level $[12,13]$. However, van Noordwijk and de Jong [14] critically pointed out that when measured at the within-species level, if individuals differ in their average resource acquisition, this allocation trade-off can be masked at the 'phenotypic' level. This is because individuals that can acquire more resources overall (either because resource acquisition is genetically or environmentally determined) will be able to allocate more, in absolute terms, to both current and future reproduction compared to individuals who acquire fewer resources $[14,15]$. It is indeed this relative balance between acquisition and allocation that will determine the appearance of this tradeoff at the phenotypic level. If there is comparatively greater variation among individuals in the allocation of resources compared to variation in acquisition, then a negative correlation among lifehistory traits is expected; if there is greater variation among individuals in the acquisition of resources compared to variation in allocation, a positive correlation can emerge ([14]; Fig 1). This has been a topic of considerable discussion in life-history theory since the 1980 s $[14,16,17]$. It appears however that explicit consideration of this balance, and the role that behavioral expression plays in it, was lost in translation during the development of the modern POLS framework (Box 1: A historical perspective).

The modern POLS framework is based on the assumption that selection has favored correlated suites of life-history and behavioral traits to resolve the trade-off between current and future reproduction and that behaviors help mediate this key trade-off [2]. This builds on the hypothesis that 
individuals that allocate more in current reproduction need a faster metabolic engine requiring more resources for fuel [18]. Individuals with different allocation strategies are predicted to also exhibit differences in behavior and hence resource acquisition abilities, with 'fast' phenotypes (greater expression of boldness, activity, exploration, aggression; see Fig 1 in [4]) acquiring more resources compared to 'slow' phenotypes. What this means is that the POLS framework is assuming the presence of among-individual variation in both resource allocation and resource acquisition, simultaneously. The hypothesis, as it stands now, is not clear about whether we expect an individual's behavior to play a larger role in their allocation or their acquisition of resources and just as importantly, what we expect the relative balance between among-individual variation in allocation and acquisition to be in a particular system. Therefore, the apparent lack of empirical support for the modern POLS framework may not be because the inherent within-individual trade-offs are absent or because behavior plays no role in them. Instead, the POLS framework has not defined its assumptions clearly enough to know exactly how the structure of trait correlations will appear at different biological levels and under different environmental conditions. Such clarity will require a better understanding of the relative balance between variation in resource allocation to resource acquisition within a particular study system and then explicit consideration of how we expect individual behavior to be linked to both these processes.

\section{POLS at different levels of biological variation}

The relative magnitude of variation in resource allocation compared to in resource acquisition will determine the strength and sign of the observed relationships between costly traits. Comparative work has shown that if species' estimated allocation to current versus future reproduction was plotted, a negative relationship would emerge whereby 'slow' species have longer lives and produce relatively fewer offspring compared to 'fast' species that exhibit quicker maturation and production of many offspring $[12,13,19,20]$. While other axes of life-history variation have been proposed $[21,22]$, this 'fast-slow' continuum explains $70 \%$ of the variation in life history strategies among species in animals [13]. 

integration at the within-species level (Box 2: Empirical testing across biological levels). The fundamental within-individual trade-off between current and future reproduction is expected to exist at this level: individual animals do not reproduce at a maximal rate immediately at birth and continue to do so throughout their lives. However, for the expected negative correlation among traits to be measurable at the within-species level (i.e., within-population among-individual level), the variation in allocation strategies among individuals would have to be larger than the among-individual variation in resource acquisition (Fig. 1). Many traits will influence both an individual's acquisition of resources and their allocation of those resources to the different fitness goals that animal needs to achieve. For example, resource allocation strategies will be determined by the coordination of many anatomical and physiological traits (e.g. organ size, hormone receptors, metabolism) [23-25]. These traits, particularly the endocrine system, have been suggested to constrain the allocation of resources resulting in well-conserved patterns of covariation among life-history traits [25]. Resource acquisition will certainly be influenced by the anatomy and physiology of an animal but also by cognitive and behavioral traits that will ultimately determine an individual's ability to locate and harvest resources $[26,27]$. It may be unlikely that a single species would harbor the same order of magnitude of variation in critical anatomical/physiological traits associated with resource allocation among individuals as would be present across a large range of species [28]. Indeed, the fast-slow continuum seems to explain a decreasing amount of life-history variation when approaching the individual level from higher biological levels [5]. If, at the within-species level, there is in fact greater relative among-individual variation in resource acquisition compared to that of resource allocation, this could mask the fundamental within-individual trade-off (Fig 1) and help explain the lack of robust empirical support for the predictions of the modern POLS framework $[9,10]$. Although quantifying variation in resource 127 acquisition and resource allocation for a given species will carry its own challenges, this information will pave the way for the refinement of the POLS framework to better understand when and at which 
biological level of variation (e.g. among-species versus among-individuals) we expect to detect the

130 fundamental trade-offs.

131

132

133

\section{Behavior's role in mediating acquisition and allocation}

After considering the relative balance between allocation and acquisition in a system, the next step in the refinement of the POLS framework will be to consider whether and how we expect behavior, and which behaviors, to be linked to either (or both) of these processes. Hormones, metabolic physiology, and immune function can play key functional roles in the allocation of resources to reproduction $[24,29]$. Therefore, if individual behavioral variation is linked to variation in the allocation of resources, then we should expect to find associations between these physiological mechanisms and behavior as well. Metabolic rate, for example, has been assumed to be one of the key physiological traits driving variation in life-history at the among-species level with 'fast' species exhibiting higher metabolic rates [30, but see 31]. The POLS framework has extended this notion to the within-species (and withinpopulation) level. However, theoretical work has shown that the associations between behavior and metabolic rate at the within-species level can be predicted to be positive, null (i.e. not correlated) or negative, depending on the energy allocation model that is used [18,32-34]. For instance, some energy allocation models predict that behavior will be controlled by bottom-up physiology and so will be just one of many traits competing for resources; indeed a recent meta-analysis found greatest support for such 'performance' models [35]. Finally, the current collective evidence does not support an association between metabolic rate and behavior at the among-individual level [8]. Altogether, this suggests that it is unlikely for behavior to play a causal role in determining the allocation of resources; understanding the mechanisms underlying behavioral expression and resource allocation would help to clarify this association. Therefore the key, unresolved question is whether behavior (and which behaviors) causally affects allocation to reproduction via metabolism (or other physiological pathways), or whether behavior is more likely to be one of the passengers, rather than the captain, of the ship when it comes to energy allocation to reproduction $[8,35]$. 

must "behave" (e.g. search, sample, move) in order to gain energy. Indeed, optimality models of energy acquisition played a large role in initiation of behavioral ecology as a study field (i.e. optimal foraging theory; [36]). All else being equal, differences in resource acquisition can then increase an individual's quality through, for example, increased growth rates or lipid storage [3]. This could allow increased allocation to several traits in absolute terms, leading to a positive correlation between costly traits at the among-individual level [15] (Fig 1). For example, in male three-spine sticklebacks (Gasterosteaus aculeatus) which perform parental care, a trade-off between nest care and nest defense is expected as both traits are costly. However, Stein and Bell [37] instead found a positive correlation whereby some male sticklebacks exhibited both greater nest care and greater defense compared to other males. These males also occupied higher quality territories suggesting that these males were intrinsically of higher quality and so could better gain such a territory, or once their territory was established, access to the resources on that territory increased the individual's overall quality. There is now considerable evidence that more active, aggressive and/or explorative individuals (i.e., 'fast' phenotypes) are able to acquire more resources (or acquire territories holding more resources) [reviewed in 3,38]. Therefore, the current evidence suggests a stronger direct functional link between behavior and resource acquisition as compared to between behavior and resource allocation. Importantly, even though the current POLS hypothesis assumes that many behaviors, e.g. boldness, aggression, exploration, activity, parental care, sociability, are integrated into POLS [4], it is not clear which behaviors actually are functionally associated with resource acquisition, allocation and/or life-history and whether we should expect the same directionality in all the relationships. Moreover, the functional role of a specific behavior probably depends on both the species (i.e. biology) and/or the environment (i.e. ecology) [39]. Thus, a major goal moving the field forward will be to

177 determine not just whether behavior is linked with life-history trade-offs, but exactly which behaviors 178 and in which environments this linkage is most likely to exist. 
If individual behavioral variation is more likely to be functionally linked to resource acquisition, and there is greater variation among individuals in resource acquisition compared to allocation, then this can mask or reverse the expected negative correlation between behavior and other life-history traits at the among-individual level (Fig 1). The current POLS framework is built on the assumption that individuals that express 'fast' phenotypes should suffer higher mortality [2-4]. But if behaviors expressed by fast phenotypes allows individuals to harvest more resources then this could compensate for the potential survival costs associated with such behavior. Indeed, some state-dependent models predict that individuals in relatively good condition or that are structurally larger are better able to avoid predation [40,41]. Moreover, a recent meta-analysis demonstrated that riskier individuals, contrary to POLS predictions, had higher survival, but only when measured in wild conditions, where presumably individuals could better express variation in their resource acquisition abilities compared to lab conditions and where extrinsic mortality plays a role [10]. Finally, classic life-history trade-offs are more likely to be revealed when animals are measured in stressful, e.g. resource-depleted, conditions [11], indicating that trade-offs can be masked by acquisition. While the presence of variation in energy acquisition is briefly mentioned in the modern POLS literature as one explanation for the absence of a trade-off between current and future reproduction [39], it does not appear that the field has comprehensively considered how the relative importance of acquisition versus allocation will modulate predictions of trait associations. Indeed, these considerations go beyond POLS and behavior as they raise questions on how best to measure any type of trade-off at different levels of biological organization. If within-individual constraints, e.g. the resources an individual allocates to current versus future reproduction, can be masked by among-individual differences in other traits, e.g. resource acquisition via behavioral expression, then this will make the observation of any expected negative relationship between traits very difficult at the among-individual level. Indeed, this is the most frequent explanation for the non-detection of reproductive costs [15], and likely contributes to problems with the phenotypic gambit [42] and comparisons between populations. Such considerations are critical though, and here we show how they can help researchers to make much-needed 
predictions about under which conditions negative versus positive relationships between life-history traits and behavior are expected to be observed (Fig 1).

\section{Concluding Remarks}

The modern pace-of-life syndrome framework can still learn important lessons from revisiting classic life-history theory while also placing more emphasis on developing formal modeling [43] and carrying out more experimental work, to push the field forward. Considering the relative balance of amongindividual variation in resource allocation compared to resource acquisition in a system, and how we expect behavioral variation to be linked to either or both of these processes, is a good first step

213 (Outstanding Questions). Such considerations have the potential to transform the modern pace-of-life syndrome framework into a more predictive framework about the integration of life-history, physiological and behavioral traits, and go even beyond POLS, providing clarifications for general lifehistory research.

\section{ACKNOWLEDGEMENTS}

We thank Denis Realé, Kimberly Mathot, Marco Festa-Bianchet and three anonymous reviewers for constructive feedback on earlier versions of the manuscript, particularly with Kim's help in refining Figure 1. 


\section{Outstanding questions}

Within a species, what is the magnitude of among-individual variation in allocation? Do aspects of a species' ecology or biology predict whether allocation or acquisition will exhibit greater variation? For example, are species that exhibit variation in mating strategies (e.g. sneaker versus territorial males) more likely to exhibit greater variation in allocation strategies? Or are species that live in resourcepoor (e.g. deserts) versus resource-rich (e.g. tropical forests) environments more or less likely to show greater variation in resource acquisition?

Are traits correlated due to constraints imposed by developmental plasticity or shared pathways (e.g. physiology, pleiotropy)? Experimental manipulations of physiological traits can be used to test for correlated responses in behavior and life-history. For example, in poikilothermic animals, will increasing metabolic rate (e.g. by manipulating temperature) result in 'fast' life-histories and 'riskier' behavior? Will artificial selection experiments with direction selection on certain physiological, behavioral or life-history traits result in correlated responses in other traits?

How can we use environmental manipulations to test POLS predictions, e.g., by constraining individuals' abilities to acquire resources within a laboratory environment? How widespread is the existence of genotype by environment interactions in allocation and acquisition strategies? Is the relationship between behavior and allocation and/or acquisition stable throughout an animal's life? How is behavioral plasticity related to POLS; do individuals with relatively 'slow' phenotypes exhibit relatively greater behavioral plasticity?

How often do POLS patterns at the additive genetic level resemble patterns at the unpartitioned phenotypic level? What is the role of permanent environmental effects (e.g., due to developmental plasticity from early life environmental conditions) in the expression of POLS? Could the mixed support 
for the POLS hypothesis be related to mismatch in trait correlations generated by permanent environmental and additive genetic effects?

\section{Box 1. A historical perspective}

The modern POLS framework $[2,4]$ is built on classical $r / K$-selection theory [44-46]. The latter postulates that phenotypes or genotypes characterized by fast growth, early maturation and rapid reproduction are selected for at low population densities while the opposite phenotypes/genotypes are favored at high population densities [45]. The modern pace-of-life framework differs from the $\mathrm{r} / \mathrm{K}$ selection framework in that it i) includes more traits (behavior and physiology) and ii) focuses exclusively on variation among individuals within populations. Thus, the POLS hypothesis integrates life-history, evolutionary biology, behavioral ecology and animal physiology research under one framework.

Similar to the $\mathrm{r} / \mathrm{K}$-selection framework that evolved through time to be more complex and realistic [45], the POLS framework has also seen important development towards acknowledging the biological and ecological complexity in the expression of pace-of-life syndromes [2,39]. One of the key differences in the development of the $\mathrm{r} / \mathrm{K}$-selection and the POLS framework is that the former is strongly linked to population dynamics and ecology, and has evolved through formal mathematical modeling while the latter is largely based on verbal models inspired by formal slow-fast continuum hypotheses $[43,45]$ and population dynamics or strong ecological elements were only involved in very recent, mostly verbal, models $[39,47,48]$. The lack of formal models might be one of the reasons for the phenomenological nature of the empirical work testing the POLS framework: solid testable predictions about the conditional expression of pace-of-life syndromes are still largely missing. The general scarcity of formal models leads to 1 ) few detailed predictions that may enable empiricists to discriminate between environmental-specific processes and 2) limited ability for empiricists to match their study system and experimental designs to particular model assumptions [43]. The lack of formal 
models in pace-of-life syndromes research is in line with a general declining trend in formal models in life-history related research [49]. Life-history research has also become more fragmented through time, potentially reducing the exchange of ideas across closely related sub-fields [49].

\section{Box 2: Empirical testing across biological levels}

The POLS framework posits that past selection have favored particular associations among traits [4]. How do we best identify these patterns and test their evolutionary and ecological implications? The signatures of past selection pressures are best identified at the additive genetic level, and assessing genetic constraints (or lack thereof) can provide information about the short-term evolutionary potential of trait correlations [11,50]. Quantitative genetic breeding designs coupled with appropriate statistical models (e.g. 'animal models' [51]) allow researchers to partition phenotypic variance among its additive genetic, permanent environmental and/or maternal components. Using such methods, Santostesfano et al. [52] found a positive genetic correlation between developmental time and lifespan in field crickets (Gryllus bimaculatus) as expected by POLS predictions; however this effect was masked at the phenotypic level due to a negative correlation imposed by permanent environmental effects. Boulton et al. [53] used similar quantitative genetic designs to test for correlations in social dominance and growth rates in swordtails (Xiphophorus birchmanni); here, the underlying genetic associations did largely follow the patterns occurring at the among-individual level. These two empirical studies illustrate how trait correlations can differ between biological levels. Accordingly, empiricists must design experimental studies that test for POLS at the appropriate biological level for the question at hand [16]. Ideally, empiricists should also plan to test POLS at multiple biological levels, as each level will provide essential insights to further our understanding of POLS and its underlying mechanisms.

Permanent environmental effects can alter the appearance of trait correlations within an animal's lifetime. Manipulating early-life environments would allow researchers to better investigate the role of developmental plasticity in generating or breaking trait associations; a promising area of empirical research that is still building a formal modeling foundation [5]. Importantly, the expression 
305 of genetic correlations itself is not immune to environmental effects $[11,54,55]$. Genetic correlations

306 can differ, for example, across environments, across samples within same population, between ages,

307 between animal groups, between populations, between species and between laboratory and wild 308 environments [11]. The mechanisms for such "instability" of genetic correlations include, for example, 309 variation in gene frequencies within and across animal groups and genotype by environment 310 interactions [11]. Therefore, a better understanding of the ecological and evolutionary factors affecting 311 expression of POLS would require studies where genetic correlations are estimated at multiple 312 different environments [56]. 
Acquisition: Collection and consumption of resources from the environment.

Allocation: Division of resources between two or more physiological processes within an individual.

Among-individual variation: Variation in a labile trait, e.g. behavior, in a sample of individuals caused by individual differences in their average trait expression (measured across multiple observations). This variation is caused by a combination of additive genetic effects and (permanent) effects of the environment on phenotypes. This level differs from the phenotypic level (see below) in that the residual variance is not included.

Biological levels: Trait expression varies across hierarchical levels of biological organization. For example, behavioral expression can vary among observations (within individuals), among individuals, among genotypes, among populations and among species.

Fast phenotype: Correlated suite of traits typically characterized by fast growth, quicker time to maturity and shorter lifespans. These individuals are predicted to also express relatively high activity, boldness, exploration and aggression and have higher metabolic rates.

Phenotypic level: Variation among phenotypes expressed within a population and that has not been partitioned among the contributing components. This level of variation will include the influence of additive genetic, permanent environmental and/or residual effects on trait expression.

Slow phenotype: Correlated suite of traits typically characterized by slower growth, delayed maturation and longer lifespan. These individuals are also predicted to express relatively low activity, boldness, exploration and aggression and have lower metabolic rates.

Within-individual trade-off: Constraint within an individual (i.e., intra-individual); typically mediated by physiological processes. For example, division of limited resources between two "competing" 
335 physiological processes, underpinning investment between current (e.g. allocation to offspring) and 336 future (e.g. allocation to growth) reproduction.

337

338 
1 Dingemanse, N.J. and Wolf, M. (2010) Recent models for adaptive personality differences: a review. Philosophical Transactions of the Royal Society B: Biological Sciences 365, 3947-3958

2 Dammhahn, M. et al. (2018) Pace-of-life syndromes: a framework for the adaptive integration of behaviour, physiology and life history. Behav Ecol Sociobiol 72, 62

3 Stamps, J.A. (2007) Growth-mortality tradeoffs and 'personality traits' in animals. Ecology Letters 10, 355-363

4 Réale, D. et al. (2010) Personality and the emergence of the pace-of-life syndrome concept at the population level. Philosophical Transactions of the Royal Society B: Biological Sciences 365, 4051-4063

5 Stearns, S.C. and Rodrigues, A.M.M. (2020) On the use of "life history theory" in evolutionary psychology. Evolution and Human Behavior DOI: 10.1016/j.evolhumbehav.2020.02.001

6 Figueredo, A.J. et al. (2013) The measurement of human life history strategy. Personality and Individual Differences 55, 251-255

7 Rushton, J.P. (1985) Differential K theory: The sociobiology of individual and group differences. Personality and Individual Differences 6, 441-452

8 Niemelä, P.T. and Dingemanse, N.J. (2018) Meta-analysis reveals weak associations between intrinsic state and personality. Proceedings of the Royal Society B: Biological Sciences 285, 20172823

9 Royauté, R. et al. (2018) Paceless life? A meta-analysis of the pace-of-life syndrome hypothesis. Behav Ecol Sociobiol 72, 64

10 Moiron, M. et al. (2020) Individual differences in behaviour explain variation in survival: a meta-analysis. Ecology Letters 23, 399-408

11 Stearns, S.C. (1992) The Evolution of Life Histories, Oxford University Press.

12 Promislow, D.E.L. and Harvey, P.H. (1990) Living fast and dying young: A comparative analysis of life-history variation among mammals. Journal of Zoology 220, 417-437

13 Healy, K. et al. (2019) Animal life history is shaped by the pace of life and the distribution of age-specific mortality and reproduction. Nature Ecology \& Evolution 3, $1217-1224$

14 van Noordwijk, A.J. and de Jong, G. (1986) Acquisition and allocation of resources: their influence on variation in life history tactics. The American Naturalist 128, 137-142

15 Reznick, D. et al. (2000) Big houses, big cars, superfleas and the costs of reproduction. Trends in Ecology \& Evolution 15, 421-425

16 Stearns, S.C. (1989) Trade-Offs in Life-History Evolution. Functional Ecology 3, 259-268

17 Reznick, D. (1985) Costs of reproduction: an evaluation of the empirical evidence. Oikos 44, 257-267

18 Careau, V. et al. (2008) Energy metabolism and animal personality. Oikos 117, 641653

19 Ricklefs, R.E. (2000) Density dependence, evolutionary optimization, and the diversification of avian life histories. Condor 102, 9-22

20 Wiersma, P. et al. (2007) Tropical birds have a slow pace of life. PNAS 104, 93409345

21 Colchero, F. et al. (2016) The emergence of longevous populations. PNAS 113, E7681-E7690 
22 Salguero-Gómez, R. et al. (2016) Fast-slow continuum and reproductive strategies structure plant life-history variation worldwide. PNAS 113, 230-235

23 Ricklefs, R.E. (1991) Structures and transformations of life histories. Functional Ecology 5, 174-183

24 Harshman, L.G. and Zera, A.J. (2007) The cost of reproduction: the devil in the details. Trends in Ecology \& Evolution 22, 80-86

25 Ricklefs, R.E. and Wikelski, M. (2002) The physiology/life-history nexus. Trends in Ecology \& Evolution 17, 462-468

26 Stephens, D.W. and Krebs, J.R. (1986) Foraging Theory, Princeton University Press.

27 Real, L.A. (1991) Animal choice behavior and the evolution of cognitive architecture. Science 253, 980-986

28 White, C.R. and Seymour, R.S. (2004) Does basal metabolic rate contain a useful signal? Mammalian BMR allometry and correlations with a selection of physiological, ecological, and life-history variables. Physiological and Biochemical Zoology 77, 929-941

29 Zera, A.J. and Harshman, L.G. (2001) The physiology of life history trade-offs in animals. Annual Review of Ecology and Systematics 32, 95-126

30 Brown, J.H. et al. (2004) Toward a metabolic theory of ecology. Ecology 85, 17711789

31 Glazier, D.S. (2015) Is metabolic rate a universal 'pacemaker' for biological processes? Biological Reviews 90, 377-407

32 Mathot, K.J. and Dingemanse, N.J. (2015) Energetics and behavior: unrequited needs and new directions. Trends in Ecology \& Evolution 30, 199-206

33 Careau, V. and Garland, T. (2012) Performance, personality, and energetics: correlation, causation, and mechanism. Physiological and Biochemical Zoology 85, 543571

34 Sih, A. et al. (2015) Animal personality and state-behaviour feedbacks: a review and guide for empiricists. Trends in Ecology \& Evolution 30, 50-60

35 Mathot, K.J. et al. (2019) The covariance between metabolic rate and behaviour varies across behaviours and thermal types: meta-analytic insights. Biological Reviews 94, 10561074

36 Pyke, G.H. et al. (1977) Optimal foraging: a selective review of theory and tests. The Quarterly Review of Biology 52, 137-154

37 Stein, L.R. and Bell, A.M. (2015) Consistent individual differences in paternal behavior: a field study of three-spined stickleback. Behav Ecol Sociobiol 69, 227-236

38 Biro, P.A. and Stamps, J.A. (2010) Do consistent individual differences in metabolic rate promote consistent individual differences in behavior? Trends in Ecology \& Evolution $25,653-659$

39 Montiglio, P.-O. et al. (2018) The pace-of-life syndrome revisited: the role of ecological conditions and natural history on the slow-fast continuum. Behav Ecol Sociobiol 72,116

40 McElreath, R. and Strimling, P. (2006) How noisy information and individual asymmetries can make 'personality' an adaptation: a simple model. Animal Behaviour 72, $1135-1139$

41 Luttbeg, B. and Sih, A. (2010) Risk, resources and state-dependent adaptive behavioural syndromes. Philosophical Transactions of the Royal Society B: Biological Sciences 365, 3977-3990

42 Willis, J.H. et al. (1991) Can one predict the evolution of quantitative characters without genetics? Evolution 45, 441-444

43 Mathot, K.J. and Frankenhuis, W.E. (2018) Models of pace-of-life syndromes (POLS): a systematic review. Behav Ecol Sociobiol 72, 41 
44 MacArthur, R.H. and Wilson, E.O. (1967) The Theory of Island Biogeography, Princeton University Press.

45 Reznick, D. et al. (2002) r-and K-selection revisited: the role of population regulation in life-history evolution. Ecology 83, 1509-1520

46 Pianka, E.R. (1970) On r- and K-Selection. The American Naturalist 104, 592-597

47 Wright, J. et al. (2019) Life-history evolution under fluctuating density-dependent selection and the adaptive alignment of pace-of-life syndromes. Biological Reviews 94, 230-247

48 Salzman, T.C. et al. (2018) Energetic trade-offs and feedbacks between behavior and metabolism influence correlations between pace-of-life attributes. Behav Ecol Sociobiol 72, 54

49 Nettle, D. and Frankenhuis, W.E. (2019) The evolution of life-history theory: a bibliometric analysis of an interdisciplinary research area. Proceedings of the Royal Society B: Biological Sciences 286, 20190040

50 Lynch, M. and Walsh, B. (1998) Genetics and Analysis of Quantitative Traits, Sinauer Associates Incorporated.

51 Wilson, A.J. et al. (2010) An ecologist's guide to the animal model. Journal of Animal Ecology 79, 13-26

52 Santostefano, F. et al. (2017) Behavioural mediators of genetic life-history trade-offs: a test of the pace-of-life syndrome hypothesis in field crickets. Proceedings of the Royal Society B: Biological Sciences 284, 20171567

54 Boulton, K. et al. (2018) Phenotypic and genetic integration of personality and growth under competition in the sheepshead swordtail, Xiphophorus birchmanni. Evolution 72 , 187-201

54 Hoffmann, A.A. and Merilä, J. (1999) Heritable variation and evolution under favourable and unfavourable conditions. Trends in Ecology \& Evolution 14, 96-101

55 Charmantier, A. and Garant, D. (2005) Environmental quality and evolutionary potential: lessons from wild populations. Proceedings of the Royal Society B: Biological Sciences 272, 1415-1425

56 Sgrò, C.M. and Hoffmann, A.A. (2004) Genetic correlations, tradeoffs and environmental variation. Heredity 93, 241-248 


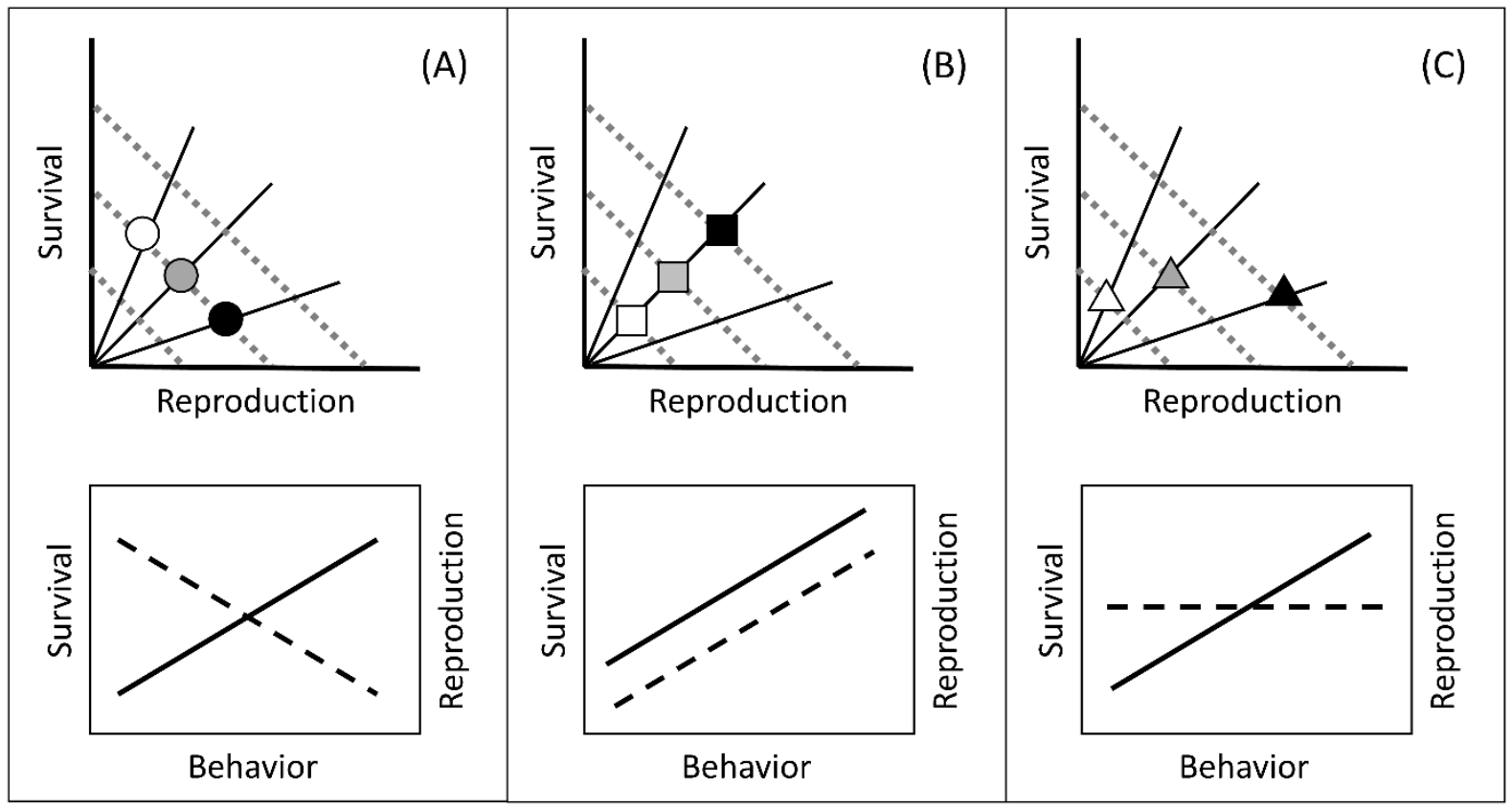

Figure 1.

473 The relationship between behavior and resource allocation versus resource acquisition can generate

474 different correlations between behavior and survival and between behavior and reproduction. Top graphs show a potential trade-off between survival and reproduction (though this could be generalized to other costly life-history traits as well); dotted lines indicate different levels of resources available

477 for allocation (driven by resource acquisition) whereas solid lines indicate differential allocation into 478 either trait. Shape color indicates individual behavior with darker colors indicating greater expression, 479 'fast' phenotypes (e.g. greater boldness). Bottom graphs illustrate the resulting expected correlation between behavior and survival (dashed lines) and between behavior and reproduction (solid lines). In panel $\mathrm{A}$, all individuals have similar acquisition and among-individual variation in behavior relates most closely to variation in allocation, with e.g. bolder individuals allocating more into reproduction at the cost of survival, as implicitly assumed in the original pace-of-life-syndromes hypothesis. In panel B, among-individual variation in behavior is most closely related to variation in resource acquisition so that bolder individuals have more resources to allocate to both survival and reproduction. In panel C, 
487 allocation whereby bolder individuals reproduce more but with no apparent survival costs. Figure 488 inspired from [14]. 